\title{
Melting of anisotropic colloidal crystals in two dimensions
}

\author{
C Eisenmann ${ }^{1}$, P Keim, U Gasser and G Maret \\ Department of Physics, University of Konstanz, 78457 Konstanz, Germany \\ E-mail: christoph.eisenmann@uni-konstanz.de
}

\begin{abstract}
The crystal structure and melting transition of two-dimensional colloids interacting via an anisotropic magnetic dipole-dipole potential are studied. Anisotropy is achieved by tilting the external magnetic field inducing the dipole moments of the colloidal particles away from the direction perpendicular to the particle plane. We find a centred rectangular lattice and a two-step melting similar to the phase transitions of the corresponding isotropic crystals via a quasi-hexatic phase. The latter is broadened compared to the hexatic phase for isotropic interaction potential due to strengthening of orientational order.
\end{abstract}

\section{Introduction}

Since the 1970s the melting transition in two dimensions has received a lot of attention. Kosterlitz and Thouless [1] introduced the idea that melting in two dimensions is driven by the unbinding of dislocation pairs. Halperin and Nelson [2] as well as Young [3] showed that the resulting so-called 'hexatic' phase still exhibits quasi-long range orientational order, which is destroyed in a second phase transition into the isotropic liquid phase by the dissociation of dislocations into free disclinations.

The predictions of this theory, which is referred to as the Kosterlitz-Thouless-HalperinNelson-Young melting scenario (KTHNY), were confirmed quantitatively by Zahn et al $[4,5]$ who used a 2D set-up of colloidal particles with isotropic magnetic dipole interaction controlled by an external magnetic field. We use essentially the same experimental system, but in addition we have the option to control the anisotropy of the interaction. This is achieved by tilting the field away from the direction perpendicular to the particle plane. We study the structure as well as the melting process of the resulting anisotropic crystals. By comparing our observations with the system with isotropic interaction we find a strengthening of the orientationally ordered phase.

1 Author to whom any correspondence should be addressed. 


\section{Experiment}

Monodisperse polystyrene particles [6] with a diameter of $4.5 \mu \mathrm{m}$ and a mass density of $1.5 \mathrm{~g} \mathrm{~cm}^{-3}$ are confined to the water/air interface of a hanging water droplet by gravity. The particles are superparamagnetic due to doping with $\mathrm{Fe}_{2} \mathrm{O}_{3}$ nanoparticles and, therefore, magnetic dipole moments can be induced by applying an external magnetic field $H$. As shown in [7] the interaction between the particles is precisely described by the dipole-dipole interaction, which dominates all other interactions. The cylindrical sample cell is horizontally aligned and the flatness of the interface is controlled with an accuracy of $\pm 1 \mu \mathrm{m}$ in order to achieve a uniform particle density throughout the sample cell, which has a diameter of $8 \mathrm{~mm}$. The colloids were observed through a $5 \times$ microscope objective by digital video microscopy. The size of the field of view was $840 \times 620 \mu \mathrm{m}^{2}$; usually $\sim 2000$ particles were observed, while the whole cell contained roughly 200000 particles. All measurements were carried out at room temperature. The external magnetic field $H$ was used to control the effective system temperature $1 / \Gamma \propto k_{\mathrm{B}} T /(\chi H)^{2}$, where $\chi$ is the magnetic susceptibility of a particle. Here we just want to stress the dependence on $H$ of the effective temperature. An exact definition of $\Gamma$ that includes the dependence on the anisotropy of the lattice is given in section 3.2. The anisotropy of the interaction between the particles was controlled by tilting the magnetic field $\mathbf{H}$ away from the direction vertical to the sample plane.

After equilibrating the system for several days in the crystalline state, the entire sample consisted of one monocrystalline domain. Then, the effective system temperature was increased stepwise by reducing the applied magnetic field until the liquid state was reached. After each change of $H$, the system was again equilibrated for half an hour before particle coordinates were stored for about another half an hour. This procedure was repeated for different tilting angles of $\mathbf{H}$.

The anisotropy of the observed crystal lattice was determined by extracting the lattice constants from the Fourier transform of the particle positions. In order to characterize the phase behaviour, we used the density-density correlation function

$$
g_{\mathrm{G}}\left(\left|\mathbf{r}-\mathbf{r}^{\prime}\right|\right)=\left\langle\exp \left(\mathrm{i} \mathbf{G} \cdot\left[\mathbf{r}-\mathbf{r}^{\prime}\right]\right)\right\rangle,
$$

the bond-angular correlation function

$$
g_{6}\left(\left|\mathbf{r}-\mathbf{r}^{\prime}\right|\right)=\left\langle\exp \left(\mathrm{i} \cdot 6\left[\theta(\mathbf{r})-\theta\left(\mathbf{r}^{\prime}\right)\right]\right)\right\rangle,
$$

as well as the time dependent Lindemann parameter [4]

$$
\gamma_{\mathrm{L}}(t)=\left\langle\left[\Delta \mathbf{u}_{j}(t)-\Delta \mathbf{u}_{j+1}(t)\right]^{2}\right\rangle / 2 a^{2} .
$$

$\mathbf{G}$ denotes a reciprocal lattice vector, $\mathbf{u}(\mathbf{r})$ is the particle displacement field. $\theta(\mathbf{r})$ defines the angle between a bond located at $\mathbf{r}$ and the $x$ axis. $j$ and $j+1$ are the indices of neighbouring particles, $a$ is the lattice constant and $\Delta \mathbf{u}(t)=\mathbf{u}(t)-\mathbf{u}(0)$.

The behaviour of the correlation functions in the isotropic case was predicted by KTHNY theory and was verified with an experimental set-up that was essentially the same as the one used for this work [4]. In the crystal, $g_{\mathrm{G}}(r)$ decays algebraically and $g_{6}(r)$ approaches a finite constant for large distances $r$, whereas in the hexatic phase $g_{\mathrm{G}}(r)$ decays exponentially and $g_{6}(r)$ algebraically. The critical exponents are 0.33 for $g_{\mathrm{G}}$ at melting and 0.25 for $g_{6}(r)$ at the hexatic-isotropic transition. In the isotropic liquid both correlation functions follow an exponential decay. The Lindemann parameter remains finite in the crystal and diverges in the hexatic as well as in the liquid phase. Its critical value at the melting point is $0.033[4,8]$.

It is not straightforward to extend these predictions to the case of an anisotropic system. The critical exponent of $g_{\mathrm{G}}$ depends on the elastic constants and, therefore, probably changes 

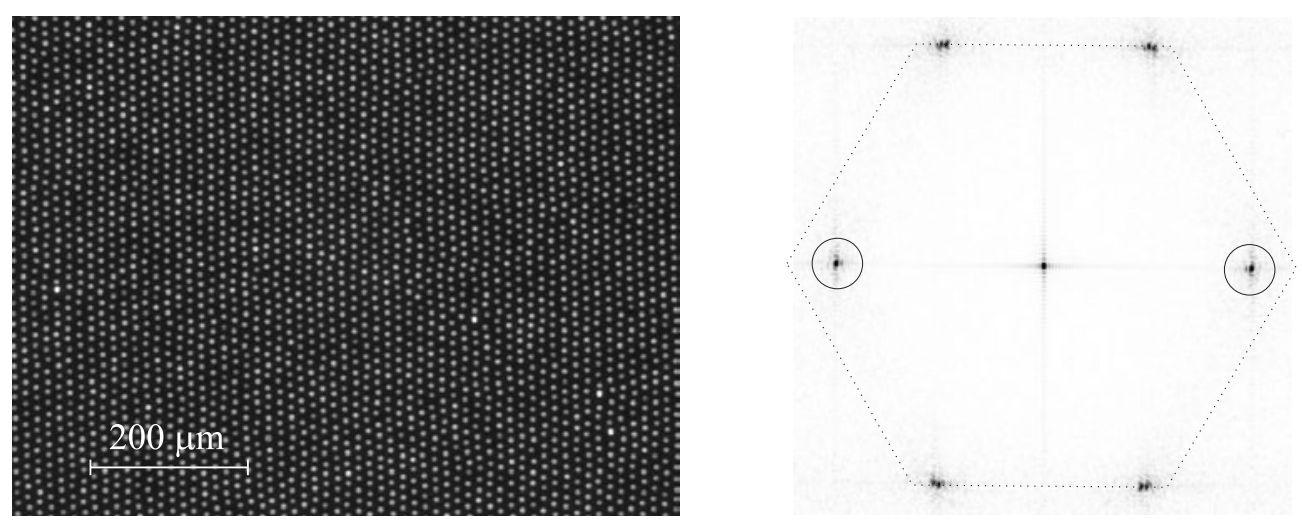

Figure 1. A camera image of an anisotropic colloidal crystal (left) and the corresponding Fourier transform of the particle coordinates (right). The centred rectangular structure can be recognized easily by comparing the peak positions to the vertices of a symmetric hexagon, which corresponds to a triangular lattice. The two peaks that are highlighted by circles were used to calculate the $g_{\mathrm{G}}$ correlation function.

in the anisotropic case. We observe a strong dependence of $g_{G}$ on the choice of $\mathbf{G}$ in the experiments and choose always the brightest Fourier peak as an input reciprocal lattice vector for the correlation function. This is one of the two shortest reciprocal lattice vectors, that are perpendicular to the in-plane field (highlighted in figure 1). As for the characterization of the orientational order, the anisotropy of the lattice influences the orientations of the nearest neighbour bonds and, therefore, complicates a direct comparison with the behaviour in the isotropic system. One might think that $g_{4}(r)$ would be the natural choice for the analysis of a centred rectangular lattice, but that would preclude a direct comparison with results on isotropic hexagonal crystals where $g_{6}(r)$ is the natural choice. To circumvent this problem, we rescale the particle positions in the direction of the in-plane component of $\mathbf{H}$ in such a way that the anisotropy disappears and the angle between adjacent bonds in the crystal becomes $60^{\circ}$. Then we calculate $g_{6}(r)$ with the rescaled coordinates and the result can be compared directly with that of the isotropic case. As far as the Lindemann parameter is concerned, the change of the long time behaviour from bound to divergent indicates in any case a melting transition.

\section{Results}

\subsection{Crystal structure}

Figure 1 shows a camera image as well as the Fourier transform of the respective particle coordinates in the case of an anisotropic interaction potential for the crystalline phase. We find a centred rectangular lattice with the shorter lattice vector $\mathbf{b}$ pointing in the direction of the in-plane component of the external magnetic field, which coincides with calculations of Froltsov et al [9]. Quantitative results for the anisotropy, defined by the ratio of the two lattice constants $a / b$, as a function of the tilting angle $\varphi$ of the magnetic field are shown in figure 2 together with the calculations from $[9,10]$. For tilting angles larger than those shown in figure 2 we find a coexistence of a centred rectangular lattice and chain-like structures and finally just chains. In our system, chain-like structures appear for smaller tilting angles than predicted in the work of Froltsov et al [9], which is to some extent due to the fact that their calculations were done at zero temperature. 


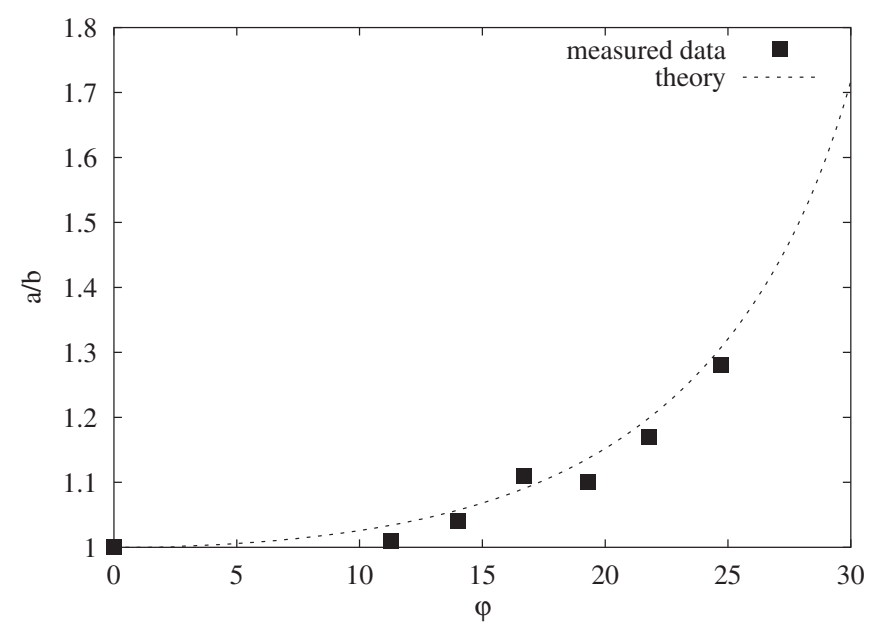

Figure 2. The ratio of the lattice constants $a$ and $b$ as a function of the tilting angle $\varphi$ of the external magnetic field (symbols: measurements; dotted curve: calculation $[9,10]$ ).

\subsection{Inverse system temperature}

The system energy of a crystalline sample depends on the crystal structure. This has to be taken into account in the definition of the effective interaction parameter ('inverse system temperature') $\Gamma=E_{\mathrm{p}} / k_{\mathrm{B}} T$, because we want to compare the melting transition of isotropic and anisotropic crystals. Here, $E_{\mathrm{p}}$ is the energy of one particle due to the interactions with all the other particles in the lattice ${ }^{2}$ :

$$
E_{\mathrm{p}}=\underset{8 \pi a^{3}}{\mu_{0}(\chi H)^{2}} \underbrace{\sum_{i=1}^{r_{i j}^{5}}}_{=C_{\text {Madelung }}(\varphi, \psi)} a^{r_{i j}^{2}-3} \underset{M}{\left(\begin{array}{l}
\mathbf{M} \\
M
\end{array} \mathbf{r}_{i j}\right)^{2}} a^{3}
$$

$\chi$ denotes the particle susceptibility, $H$ the external magnetic field, $a$ the larger of the two lattice constants, $\mathbf{M}$ is the magnetic dipole moment of a single particle and $\mathbf{r}_{i j}$ the distance vector between the particles $i$ and $j . \psi=\arccos (b / 2 a)$ represents the angle between the two lattice vectors. By expressing the factor $a^{-3}$ in equation (4) by means of the particle density $\rho=\left(2 a^{2} \cos \psi \sin \psi\right)^{-1}$ we can write

$$
\Gamma=\begin{gathered}
E_{\mathrm{p}} \\
k_{\mathrm{B}} T
\end{gathered}=\begin{gathered}
\mu_{0}(\chi H)^{2} \\
8 \pi k_{\mathrm{B}} T
\end{gathered} \rho^{3 / 2} \cdot(2 \cos \psi \sin \psi)^{3 / 2} \cdot C_{\text {Madelung }}(\varphi, \psi) .
$$

In order to calculate the latter two factors of this definition, we need to know how $\psi$ depends on the tilting angle $\varphi$. As shown in section 3.1 and figure 2, the dependence of the ratio of the lattice constants $a / b$ on $\varphi$ is known from the theory of Froltsov et al [9]. Therefore, the variation of $\psi=\arccos (b / 2 a)$ with $\varphi$ is known and we can calculate the correction to $\Gamma$ due to the anisotropy numerically. In [4] the Madelung constant was not included in the definition of $\Gamma$. As a consequence, the $\Gamma$ values for $\varphi=0$ that are presented here differ from the ones in [4] by a constant factor.

2 As shown in [9], the energy of interaction between two induced dipoles is just half as large as that between permanent dipoles which leads to the prefactor ${ }_{8 \pi}^{\mu_{0}}$ instead of ${ }_{4 \pi}^{\mu_{0}}$. 

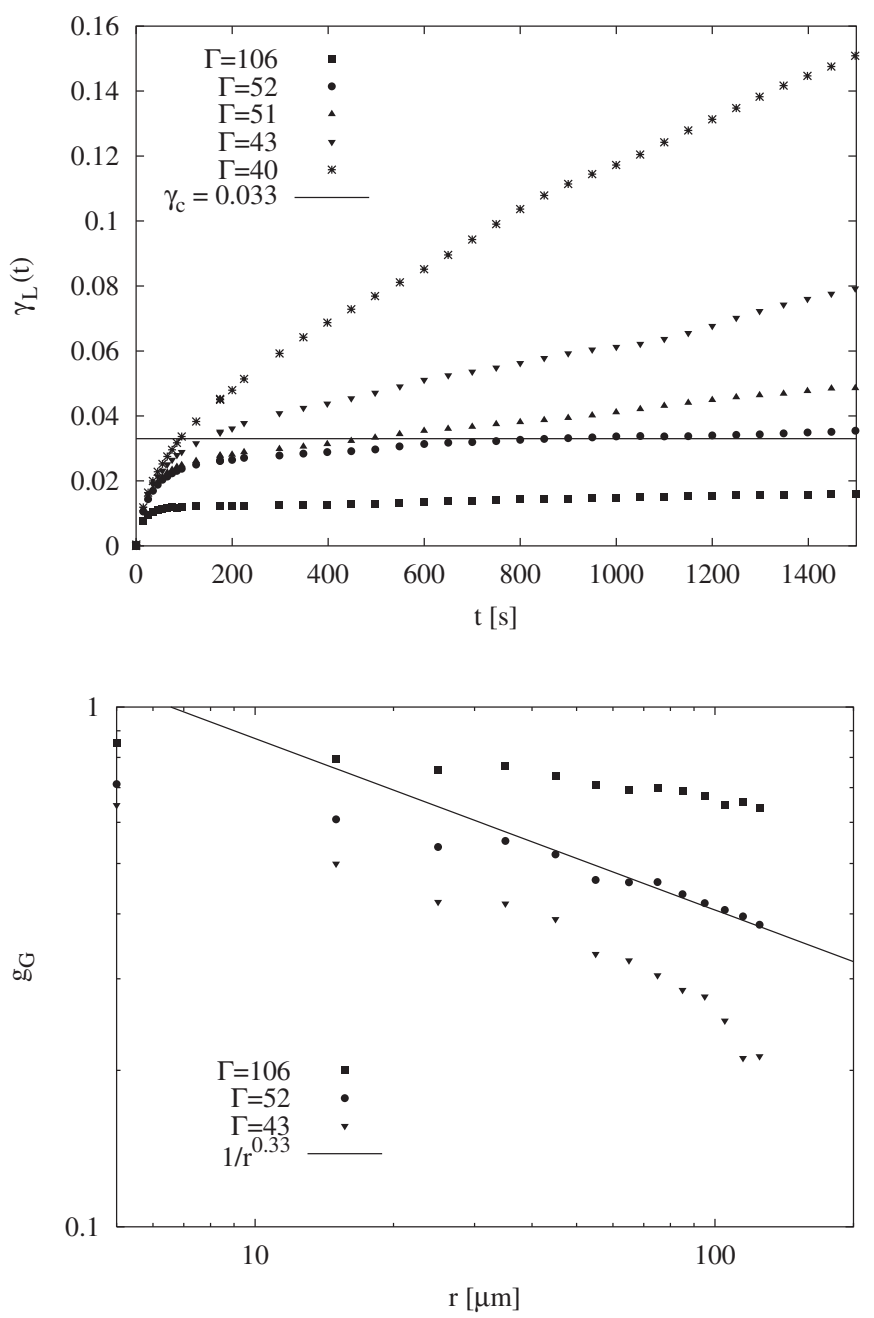

Figure 3. The time dependent Lindemann parameter for the tilting angle $\varphi=19^{\circ}$.
Figure 4. Density-density correlation functions for the tilting angle $\varphi=19^{\circ}$.

\subsection{Melting}

In figure 3 the time dependent Lindemann parameter is shown for different values of $\Gamma$ and a tilting angle $\varphi=19^{\circ}$. For $\Gamma=106$ the Lindemann parameter is bounded to values $<0.02$; at $\Gamma=52$ the critical value of 0.033 is reached at long times; and for smaller inverse system temperatures the Lindemann parameter diverges. As shown in figure 4, the density-density correlation function decays with an exponent of roughly 0.33 at $\Gamma=52$. The behaviour of both $\gamma_{\mathrm{L}}(t)$ and $g_{\mathrm{G}}(r)$ shows that melting takes place at $\Gamma_{\mathrm{m}}^{19^{\circ}}=52$, while the second transition from quasi-hexatic to liquid takes place at $\Gamma_{i}^{19^{\circ}}=41$, where $g_{6}(r)$ decays with the critical exponent of 0.25 (see figure 5). The analysis of the defects reflects exactly this behaviour. As can be seen in figure 6 , at $\Gamma=52$ several dislocation pairs as well as a few free dislocations are present in the sample. No disclinations have appeared yet, whereas in figure 7 for $\Gamma=40$ there are a large number of dislocations and several disclinations are unbound, indicating a liquid phase.

Our data lead to the following conclusions: in the case of the anisotropic interaction potential we find a two-step melting similar to the isotropic case: the quasi-long range 

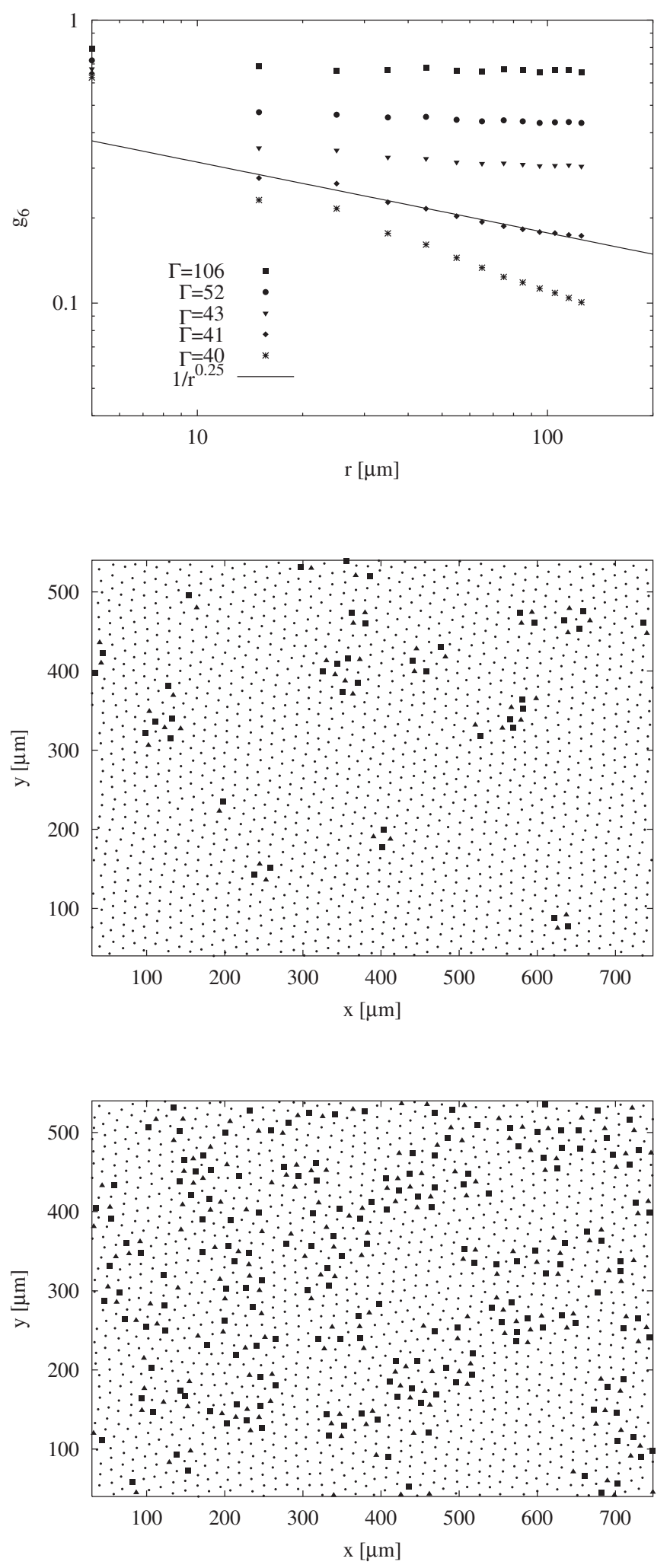

Figure 5. Bond-angular correlation functions for the tilting angle $\varphi=$ $19^{\circ}$.

Figure 6. A snapshot of a sample at $\varphi=19^{\circ}$ and $\Gamma=$ 52 close to the melting point. Each dot represents the position of a particle, where fivefoldand sevenfold-coordinated particles are highlighted with squares and triangles, respectively.

Figure 7. A snapshot of a liquid at $\varphi=19^{\circ}$ and $\Gamma=40$. The number of defects is much larger than in the crystal or hexatic state (see figure 6). As expected for a liquid, there are free disclinations, though at $\Gamma=40$ the majority are still bound in dislocations. 


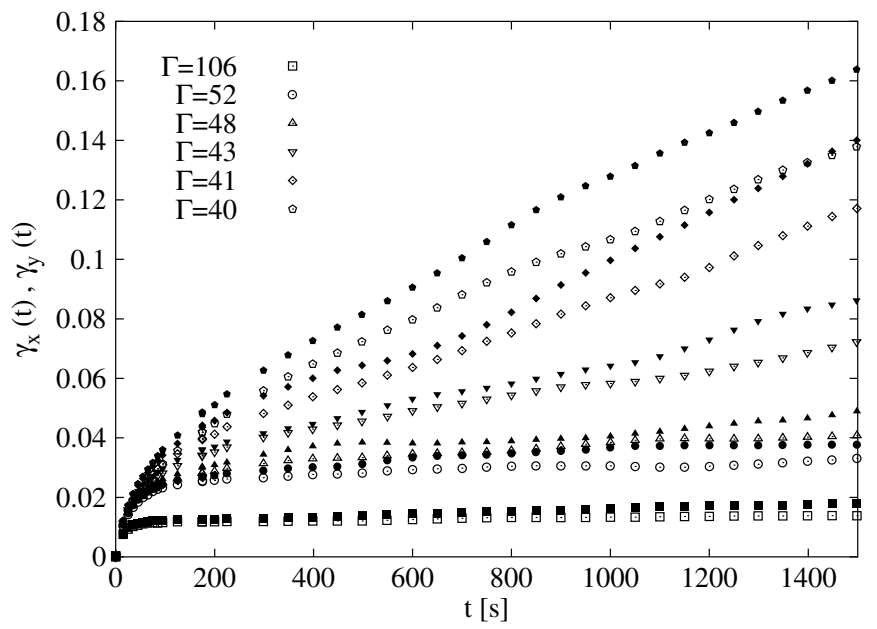

Figure 8. The Lindemann parameter parallel (open symbols) and perpendicular (full symbols) to the direction of the magnetic field for the tilting angle $\varphi=19^{\circ}$.

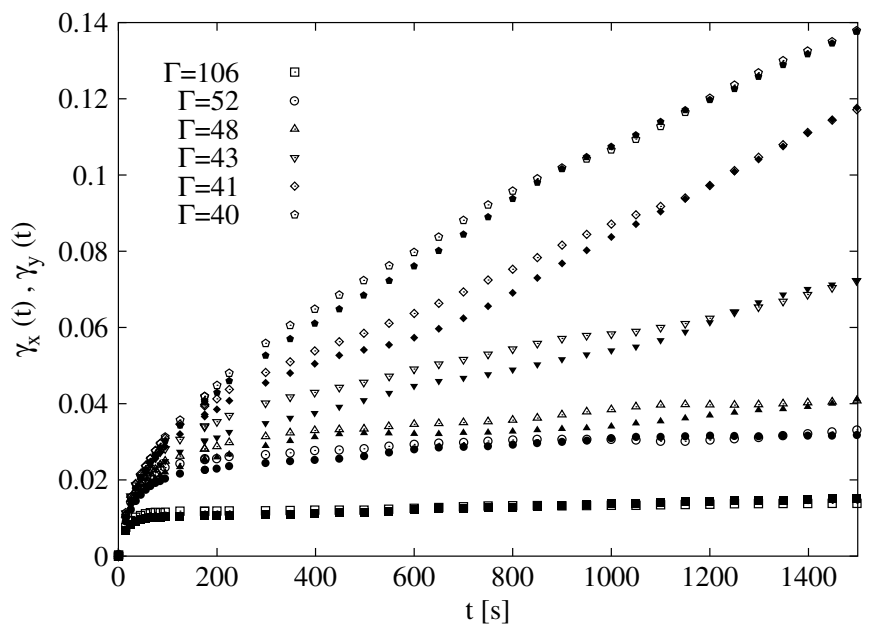

Figure 9. The same data as in figure 8, but renormalized by the anisotropy (see the text for details).

translational order is destroyed in a first phase transition by the unbinding of dislocations. The resulting phase has quasi-long range orientational order in the sense that the particle coordinates of this phase can be mapped onto a phase with hexagonal symmetry by stretching. We suggest calling this phase quasi-hexatic, emphasizing the fact that, as in the hexatic phase, translational order is short range whereas orientational order is still quasi-long range. Analogously to the isotropic case, the orientational order is lost in a second phase transition to an anisotropic liquid.

When compared to the values for the isotropic case $\left(\Gamma_{\text {melting }}=48.9\right.$ and $\Gamma_{\text {hexatic } \rightarrow \text { liquid }}=$ 46.2), our data lead to the conclusion that the quasi-hexatic phase appearing in the anisotropic melting process is enlarged in comparison with the isotropic case. The phase transition from hexatic to liquid is shifted to a lower inverse system temperature while the melting point 
remains close to that of the isotropic case. This strengthening of the orientational order can be explained by the anisotropy of the interaction. As mentioned in section 3.1, the shorter lattice vector of the centred rectangular lattice always points in the direction of the in-plane component of the external magnetic field. Consequently, the nearest neighbour bonds are aligned by the anisotropy, which facilitates orientational order down to lower values of $\Gamma$. As expected, $q_{6}=\langle\exp (\mathrm{i} 6 \cdot \theta(\mathbf{r}))\rangle$ stays finite in the liquid and, therefore, the liquid is also anisotropic.

Another effect of the anisotropy of the interaction is that the Lindemann parameter becomes anisotropic, too. This can be seen in figure 8, where $\gamma_{x}(t)=\left\langle\left[\Delta x_{j}(t)-\Delta x_{j+1}(t)\right]^{2}\right\rangle / a^{2}$ and $\gamma_{y}(t)=\left\langle\left[\Delta y_{j}(t)-\Delta y_{j+1}(t)\right]^{2}\right\rangle / a^{2}$ are plotted separately for a tilting angle $\varphi=19^{\circ}$ (with the in-plane component of the magnetic field pointing in the $y$ direction and $x$ and $y$ being the corresponding components of the displacements). However, if the anisotropy of the lattice is taken into account and the $x$ component of the Lindemann parameter is renormalized accordingly, the $x$ and $y$ components fall on top of each other, as illustrated in figure 9-at least for the long time limit.

\section{Conclusions and perspective}

We have studied the structure and the melting process for anisotropic colloidal crystals in two dimensions. We find a centred rectangular lattice with an anisotropy in quantitative agreement with theoretical predictions. Knowing the structure of the lattice, we calculate the Madelung constant, and we can use it to map the behaviour of the anisotropic system onto that of the isotropic one. For the tilting angles $\left(<20^{\circ}\right)$ of the external magnetic field studied, we observe a two-step melting via a quasi-hexatic phase, which is enlarged compared to the hexatic phase of isotropic melting, because the orientational order is strengthened by the anisotropy of the interaction. We also find an anisotropy in the Lindemann parameter which can be explained exclusively by the anisotropy of the lattice.

We intend to measure the whole phase diagram for different tilting angles, in particular for larger angles where we expect an anisotropic melting into a smectic-like phase. These data together with a detailed defect analysis will be published in a forthcoming paper.

\section{Acknowledgments}

We acknowledge fruitful discussions with D Reinke, V A Froltsov, C N Likos, and H Löwen. This work was supported by the Deutsche Forschungsgemeinschaft (DFG) within subproject C 2 of the SFB Transregio 6 programme 'Physics of colloidal dispersions in external fields'.

\section{References}

[1] Kosterlitz J M and Thouless D J 1973 J. Phys. C: Solid State Phys. 61181

[2] Nelson D R and Halperin B I 1979 Phys. Rev. D 192457

[3] Young A P 1979 Phys. Rev. B 191855

[4] Zahn K, Lenke R and Maret G 1999 Phys. Rev. Lett. 822721

[5] Zahn K and Maret G 2000 Phys. Rev. Lett. 853656

[6] Dynabeads M-450 Epoxy from www.dynalbiotech.com

[7] Zahn K, Mendez-Alcaraz J M and Maret G 1997 Phys. Rev. Lett. 79175

[8] Bedanov V M, Gadiyak G V and Lozovik Y E 1985 Phys. Lett. A 109289

[9] Froltsov V A, Blaak R, Likos C N and Löwen H 2003 Phys. Rev. E 68061406

[10] Froltsov V A 2003 private communication 